Short Research Communication

\title{
Identification of Novel Focal Adhesion Kinase Substrates: Role for FAK in NFKB Signaling
}

\author{
Sheila Figel Dwyer, Lingqiu Gao and Irwin H. Gelman ${ }^{\bowtie}$ \\ Department of Cancer Genetics, Roswell Park Cancer Institute, USA. \\ $\square$ Corresponding author: Irwin H. Gelman, Department of Cancer Genetics, Roswell Park Cancer Institute, Elm and Carlton Streets Buffalo, \\ NY 14263, 716-845-7681, Irwin.gelman@roswellpark.org.
}

(C) 2015 Ivyspring International Publisher. Reproduction is permitted for personal, noncommercial use, provided that the article is in whole, unmodified, and properly cited. See http://ivyspring.com/terms for terms and conditions.

Received: 2014.08.05; Accepted: 2014.11.10; Published: 2015.02.17

\begin{abstract}
Focal adhesion kinase (FAK) is a major signaling molecule which functions downstream of integrins or in conjunction with mitogenic signaling pathways. FAK is overexpressed and/or activated in many types of human tumors, in which it promotes cell adhesion, survival, migration and invasion. In addition to FAK's ability to regulate signaling through its scaffolding activities, FAK encodes an intrinsic kinase activity. Although some FAK substrates have been identified, a more comprehensive analysis of substrates is lacking. In this study, we use a protein microarray to screen the human proteome for FAK substrates. We confirm that several of the proteins identified are bona fide in vitro FAK substrates, including several factors which are known to regulate the NFKB pathway. Finally, we identify a role for FAK's kinase activity in both canonical and non-canonical NFKB signaling. Our screen therefore represents the first high throughput screen for FAK substrates and provides the basis for future in-depth analysis of the role of FAK's kinase activity in the processes of tumorigenesis.
\end{abstract}

Key words: FAK, substrates, phosphotyrosine, kinase, NFKB, IKKa.

\section{Introduction}

Focal adhesion kinase (FAK) is a major signaling molecule involved in the control of cell proliferation, survival, and motility. FAK functions in focal adhesions to facilitate intracellular signaling by transmembrane integrins during adhesion. In the simplest mechanism of activation, FAK is recruited to clustered integrins following cell attachment to extracellular matrix components [1]. Following recruitment, autophosphorylation on tyrosine 397 both activates FAK's kinase activity and creates a high-affinity SH2 binding site for cellular Src (c-Src) [2]. Binding to FAK in turn activates Src's kinase activity; consequently, Src phosphorylates additional tyrosines in FAK which stimulates maximal FAK kinase activity $[3,4]$.

Integrin clustering and Src recruitment serve to activate FAK's kinase activity, however little is known about the downstream effects of this. A few proteins, including paxillin [5], Shc [6], and p130Cas [7], have been shown to be phosphorylated by FAK in vitro. However, increased levels of phospho-paxillin and phospho-p130Cas are detected in FAK-null mouse embryonic fibroblasts [8], suggesting an overlap with other tyrosine kinases such as Src and the FAK family member, Pyk2 [9].

In an effort to identify FAK-specific substrates, we employed a novel in vitro approach in which a microarray with recombinant human proteins was subjected to an in situ kinase reaction with purified FAK. Among the many candidates identified, four proteins were confirmed in individual assays as novel in vitro FAK substrates. FAK was found to phosphorylate multiple molecules within the NFKB signaling pathway, including the major pathway component CHUK/IKKa, which was found to be phosphorylated by FAK both in vitro and in vivo. Further, we found that FAK kinase activity promotes both canonical and 
non-canonical NFKB signaling, partially through IKKa phosphorylation.

\section{Materials and methods}

\section{Reagents}

The following reagents/kits were used: ProtoArray Human Protein Microarray v5.0 Kinase Substrate Identification Complete Kit (Invitrogen), full-length active Src (SignalChem), GST-FAK (Invitrogen), baculovirus-encoded Pyk2 (gift of Vita Golubovskaya, Roswell Park Cancer Institute [RPCI]), purified vitronectin (Advanced BioMatrix), purified GST-PTPN5[a.a.17-565] (ProteinTech), FAK inhibitor PF-573,228 (Sigma), TNFa (Abcam), Recombinant Light (Enzo Life Sciences). The following antibodies $(\mathrm{Ab})$ were used: HA (Abm), MAb-4G10 (anti-phosphotyrosine [PTyr]) and FAK (Millipore), poY397-FAK (BD Transduction), GAPDH (Santa Cruz), p100/p52 (Cell Signaling).

\section{Cell culture}

Spodoptera frugiperda Sf-9 cells (Xinjiang Wang, RPCI), HEK293T and MCF7 (ATCC, Manassas, VA), $\mathrm{FAK}+/+$ and FAK-/- mouse embryonic fibroblasts (MEF) from a p53-/- background (T. Yamamoto and S. Aizawa, University of Tokyo), HeLa (A. Gudkov, RPCI) and MCF-7 (A. Bakin, RPCI). Sf9 were maintained in Grace's supplemented media with $10 \%$ fetal bovine serum, 1\% Pluronic F-68 and penicillin/streptomycin; cultures were grown in glass flasks shaking at $165 \mathrm{rpm}$ at $27^{\circ} \mathrm{C}$. All other cells lines were maintained in DMEM plus 10\% FBS and penicillin/streptomycin.

\section{Plasmids}

Mouse FAK cDNA was cloned into pFASTBac-HTb (Invitrogen) between BamHI and KpnI sites to form pFastFAK. GST-PDCD6 was generated by cloning the full-length open reading frame as an EcoRI fragment into pGEX-5X-2 (GE Healthcare). GST-paxillin (chicken; a.a.1-151) in pGEX-5X was described previously [5]; pCR-HA-IKKa (Addgene \#15469), pLUdR-puro-YFP-FAK-Y180A/M183A (FAK180) (gift of M. Schaller, West Virginia University), NFkB-Luciferase (NK-Luc; gift of E. Kurenova, RPCI); pCMV-Renilla (gift of A. Bakin, RPCI), pCMV4-p100 (Addgene \#23287). Y->F mutants in untagged IKKa (gift of E. Kurenova) were generated by site-directed mutagenesis using the primers msIKKa-Y187F: TGTGGGAACATTGCAGTTTTTGG CCCCAGAGCTCTTT, msIKKa-Y198F: CTTTGAAAA TAAGCCGTTCACAGCCACTGTGGATTATTGG, and msIKKa-Y500F: GAGAGATATAGTGAGCAGA TGACTTTTGGGATATCTTCAG.

\section{Baculovirus purification of FAK}

FAK bacmid DNA was generated by transformation of pFastFAK into DH10Bac ${ }^{\mathrm{TM}}$ (Invitrogen). Recombinant FAK bacmid DNA was then transfected into Sf9 using Cellfectin (Invitrogen), and these cells were used to generate high-titer baculovirus. Sf9 were infected with high-titer virus for $48 \mathrm{~h}$, pelleted by centrifugation at $1 \mathrm{~K}$ for $10 \mathrm{~min}$ at $4^{\circ} \mathrm{Cm}$ resuspended in PBS, then lysed in $2 \mathrm{X}$ lysis buffer $(20 \mathrm{mM}$ Tris-Cl, $\mathrm{pH}$ 7.4, $500 \mathrm{mM} \mathrm{NaCl}, 10 \mathrm{mM}$ imidazole, $2 \%$ Triton-X100, $2 \mathrm{mM}$ phenylmethanesulfonyl fluoride, Roche cOmplete ${ }^{\circledR}$ protease inhibitor cocktail) on ice for $30 \mathrm{~min}$. Cleared lysate was added 1:1 to equilibration buffer ( $20 \mathrm{mM}$ Tris-Cl, pH 8.0, $500 \mathrm{mM} \mathrm{NaCl}, 10$ $\mathrm{mM}$ imidazole) prior to binding to $\mathrm{Ni}^{2+}$ columns (Thermo Scientific) for $30 \mathrm{~min}$ at $4^{\circ} \mathrm{C}$. Columns were washed thrice with $20 \mathrm{mM}$ Tris-Cl, $\mathrm{pH} 8.0,500 \mathrm{mM}$ $\mathrm{NaCl}, 50 \mathrm{mM}$ imidazole, and FAK was eluted in buffer containing $500 \mathrm{mM}$ imidazole. Imidazole was removed by dialysis and His-FAK was concentrated using Amicon ${ }^{\circledR}$ Ultra (Millipore) spin columns- 125 $\mathrm{kDa}$ cutoff. FAK was stored at $-20^{\circ} \mathrm{C}$ in $50 \mathrm{mM}$ Tris-Cl, pH 7.4, $150 \mathrm{mM} \mathrm{NaCl}, 0.5 \mathrm{mM}$ EDTA, and $1 \mathrm{mM}$ DTT.

\section{Protein microarray}

The ProtoArray ${ }^{\circledR}$ Human Protein Microarray v5.0 was probed according to the manufacturer's instructions: slides blocked in 1\% BSA in PBS for $1 \mathrm{~h}$ at room temperature were incubated in Invitrogen $\mathrm{Ki}$ nase Buffer containing $10 \mu \mathrm{Ci} \gamma^{-33 P}$-ATP (Perkin-Elmer) with or without $100 \mathrm{nM}$ His-FAK for $1 \mathrm{~h}$ at $30^{\circ} \mathrm{C}$. Slides were washed twice each with $0.5 \%$ SDS and ultrapure water, dried and exposed to film. Images were scanned, saved as 16-bit TIF files and uploaded to the Protoarray Prospector Imager (Invitrogen), and using Protoarray Prospector Analyzer (Invitrogen) software, positive hits ( $>1.5$-fold over control; coefficient of variation $<0.5$, Z-factor $>0.5$ ) were identified by comparing signals within duplicate spots, measurement of signal-to-noise ratio, and comparison of signals to negative controls.

\section{Protein purification}

GST, GST-paxillin and GST-PDCD6 were purified from BL21-pLysS as described previously [10].

\section{In vitro kinase (IVK) assays}

For FAK-IPs, lysates made from HEK293T cells expressing HA-FAK were incubated with aHA at $4^{\circ} \mathrm{C}$ overnight, then with $20 \mu \mathrm{l}$ Protein A/G resin (Santa Cruz) for $1 \mathrm{~h}$. Beads were washed twice with RIPA buffer and twice with kinase buffer $(20 \mathrm{mM}$ Hepes $\mathrm{pH}$ $7.2,5 \mathrm{mM} \mathrm{MnCl} 2$, and $5 \mathrm{mM} \mathrm{MgCl}_{2}$ ), then resuspended in $20 \mu \mathrm{l}$ kinase buffer. $5 \mu \mathrm{l}$ beads were reacted with substrate protein plus $10 \mu \mathrm{Ci}{ }^{32} \mathrm{P}-\mathrm{ATP}$ at $30^{\circ} \mathrm{C}$ for 
30 min, after which samples were subjected to SDS-PAGE. Dried gels were autoradiographed. Src, FAK, and Pyk2 IVK buffer contained $50 \mathrm{mM}$ Tris-Cl $\mathrm{pH} 7.4,10 \mathrm{mM} \mathrm{MgCl} 2,1 \mathrm{mM}$ DTT, and $0.1 \mathrm{mM}$ $\mathrm{Na}_{3} \mathrm{VO}_{4}$. For non-radioactive IVK assays, HA-IKKa-beads were incubated with GST-FAK and $10 \mathrm{mM}$ ATP at $30^{\circ} \mathrm{C}$ for $30 \mathrm{~min}$, and then analyzed for phosphate incorporation by PTyr immunoblot (IB).

\section{Luciferase assays}

Cells were transfected with NK-Luc and CMV-Renilla, and stimulated with TNFa where indicated. Luciferase activity was measured using the Promega Dual-Glo Luciferase Assay System according to manufacturer instructions. In FAK-/- cells stably expressing the pLA-NFkB-Luciferase reporter +/FAK180, equal numbers of cells were assayed for luciferase activity using the Promega Luciferase Assay System according to manufacturer instructions.

\section{Results and Discussion}

\section{Identification of FAK substrates using protein microarray}

The Protoarray Human Protein Microarray, containing 9,483 purified human proteins printed in duplicate on a nitrocellulose-coated slide, was used to identify FAK substrates by incubating with either purified FAK plus ${ }^{33} \mathrm{P}-\mathrm{ATP}$, or with ${ }^{33 \mathrm{P}-\mathrm{ATP}}$ alone. The known FAK substrates, p130 Cas and Shc, were absent from the array, and two paxillin sequences listed were either incomplete or incorrectly annotated. As a control, we showed that FAK could phosphorylate GST-paxillin, but not GST, in custom microarrays (Fig. 1a, right). Normalizing to the positive control, PKCeta, which binds 33 P-ATP directly (Fig. 1b), 43 candidate FAK substrates were identified (Fig. 2a). Due to manufacturing inconsistencies (Invitrogen communication), the data from blocks 1-20 were excluded from analysis, thus limiting the analysis to 6,322 proteins.

Pubmed and Gene (http://ncbi.nlm.nih.gov) searches were used to assign functions to the identified proteins (Fig. 2). Substrates were grouped into 7 categories: Signaling (32.6\%), transcription/translation (32.6\%), tissue architecture (7\%), protein processing/turnover $(7 \%)$, mitosis $(4.7 \%)$, survival $(2.3 \%)$, and metabolism $(2.3 \%)$. The functions of 5 proteins $(11.6 \%)$ could not be assigned. The identification of many signaling proteins was expected based on FAK's role in many pathways, yet it is surprising that few of these candidates are involved in the signaling pathways known to typically cooperate with or be regulated by FAK, such as PI3K [11] and ERK/MAPK [12]. Interestingly, FAK also showed a substrate preference for molecules involved in transcription and translation, processes in which FAK has not yet been implicated. If verified, phosphorylation of such factors may suggest entirely new FAK functions.
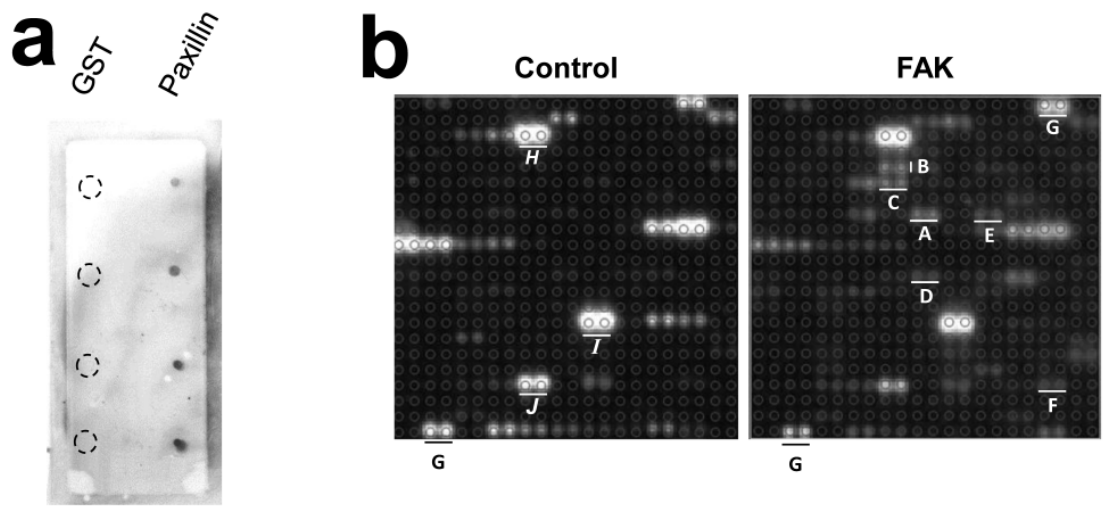

\begin{tabular}{|cccc|}
\hline & Protein & Position ID & Signal \\
A & LAMC1 & B40R08C11 & $20,291.80$ \\
B & RPL35 & B40R05C09 & $17,101.40$ \\
C & RBM8A & B40R06C09 & $14,183.00$ \\
D & ZFAND3 & B40R12C11 & $11,353.60$ \\
E & VTN & B40R08C15 & $10,944.70$ \\
F & TCF25 & B40R19C19 & $10,844.30$ \\
G & PKCeta & & \\
H & PAK4 & B40R03C09 & \\
I & ABL1 & B40R15C13 & \\
$\boldsymbol{J}$ & TAOK3 & B40R19C09 & \\
\hline
\end{tabular}

Figure 1. Microarray screen for FAK substrates. a. Left: Autoradiograph image of control and FAK protein microarray slides. Right: Control microarray containing 1, 5, 10 and $25 \mathrm{ng} /$ dot of GST or GST-Paxillin. b. Spot-pair signals from control and FAK slides block \#40 (upper panels), with identified proteins, microarray positions and relative signal intensities (average pixels/pair) below. A-F, proteins with increased phosphorylation by FAK relative to control; G, positive control protein, PKCeta; $H$-J, proteins with equal or decreased phosphorylation levels in the FAK slide compared to control. 


\begin{tabular}{|ll|}
\hline \multicolumn{2}{|c|}{ Signaling } \\
Protein & Signal \\
STK33 & $26,727.60$ \\
TNIK & $16,131.60$ \\
PTPN5 & $14,951.70$ \\
CD2BP2 & $13,212.10$ \\
GABARAP & $10,183.20$ \\
CHUK & $9,766.91$ \\
FGF1 & $9,464.33$ \\
CHERP & $9,154.44$ \\
TNIP2 & $7,284.54$ \\
6CKINE & $5,401.85$ \\
FAM82C & $4,949.35$ \\
C1QTNF1 & $4,835.37$ \\
PTPN7 & $4,148.84$ \\
MYBBP1A & $4,096.93$ \\
\hline
\end{tabular}

\begin{tabular}{|ll|}
\hline \multicolumn{2}{|c|}{ Transcription/translation } \\
Protein & Signal \\
LUC7L & $17,868.20$ \\
RPL35 & $17,101.40$ \\
RTF1 & $16,111.20$ \\
SRrp35 & $15,563.40$ \\
RBM8A & $14,183.00$ \\
SFRS6 & $12,424.80$ \\
ZFAND3 & $11,353.60$ \\
TCF25 & $10,844.30$ \\
EIF2A & $9,368.13$ \\
SUDS3 & $9,100.87$ \\
RDBP & $7,980.16$ \\
LOC81691 & $7,871.89$ \\
SRP19 & $7,401.93$ \\
WDR57 & $5,750.93$ \\
\hline
\end{tabular}

\begin{tabular}{|c|c|}
\hline \multicolumn{2}{|c|}{ Tissue architecture } \\
\hline Protein & Signal \\
\hline LAMC1 & $20,291.80$ \\
\hline VTN & $10,944.70$ \\
\hline CLDN16 & $4,384.52$ \\
\hline \multicolumn{2}{|c|}{ Processing/turnover } \\
\hline Protein & Signal \\
\hline GALNT13 & $16,383.70$ \\
\hline PSMA6 & $9,685.84$ \\
\hline USP39 & $8,825.69$ \\
\hline \multicolumn{2}{|c|}{ Mitosis } \\
\hline Protein & Signal \\
\hline PSRC1 & $17,879.50$ \\
\hline KIF2OA & $11,986.00$ \\
\hline
\end{tabular}

\begin{tabular}{|ll|}
\hline \multicolumn{2}{|c|}{ Survival } \\
Protein & Signal \\
PDCD6 & $8,391.83$ \\
\hline \multicolumn{2}{|c|}{ Metabolism } \\
\hline Protein & Signal \\
PDK3 & $25,113.50$ \\
\hline \multicolumn{2}{|c|}{} \\
\hline \multicolumn{2}{|c|}{ Unknown function } \\
Protein & Signal \\
C1orf35 & $10,740.40$ \\
DTWD1 & $8,469.72$ \\
MFAP3L & $7,615.33$ \\
ANKMY2 & $6,419.88$ \\
KIAA1539 & $6,038.96$ \\
\hline
\end{tabular}

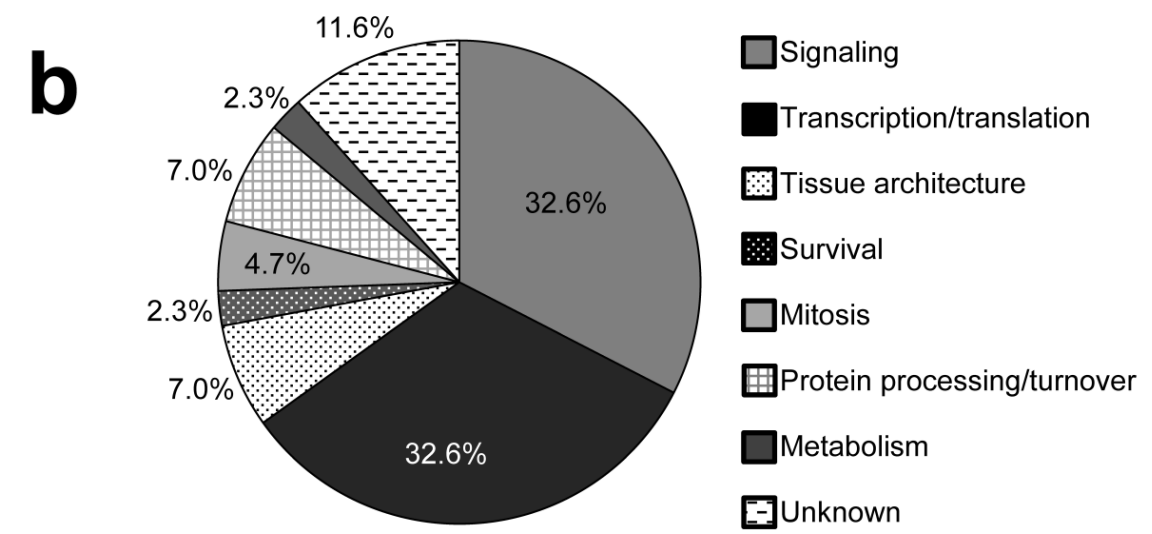

Figure 2. Candidate identified FAK substrates. a. Identified FAK substrates categorized according to function groups. b. Percentage of FAK substrates based on functional grouping.

\section{Vitronectin, PDCD6 and PTPN5 are novel FAK substrates}

In order to validate screen hits, we performed individual kinase assays with several candidate proteins known to function in pathways relevant to FAK biology. The secreted glycoprotein vitronectin (VTN) is an extracellular matrix component which plays a role in maintaining tissue architecture by mediating interactions between cells and the extracellular matrix. Two other proteins identified, Programmed cell death 6 (PDCD6) and Protein tyrosine phosphatase non-receptor type 5 (PTPN5), modulate cell survival through the regulation of apoptosis. PTPN5 was shown to positively regulate apoptosis through its dephosphorylation of Bak [13]; PDCD6 has been shown to promote apoptosis in ovarian cancer cells [14]. Vitronectin, PDCD6 and PTPN5 were phosphorylated in FAK-IVK reactions (Fig. $3 a-c)$. Src phosphorylated PDCD6 and PTPN5, although to lesser extents than FAK, and indeed, when normalized to Src or FAK autophosphorylation levels, PTPN5 seems to be a better substrate of FAK. In contrast, Pyk2 was unable to phosphorylate either protein in vitro (Fig. $3 \mathrm{~d}-\mathrm{e}$ ) although it could phosphorylate paxillin. This suggests a degree of specificity for FAK within the FAK/Pyk2 kinase family. The relevance of vitronectin phosphorylation is less clear because these proteins are not found in the same cellular compartment (FAK: plasma membrane; vitronectin: extracellular matrix). However, at least two phosphotyrosine sites on vitronectin have been identified in cell/tissue lysates (http://www.phosphosite.org). It is unclear why FAK phosphorylates vitronectin, PDCD6 or PTPN5 with different efficiencies, yet we have noted that once normalized for equal protein concentrations, FAK phosphorylates many full-length proteins, such as paxillin, to a lesser extent than truncated versions of the substrates (e.g.- paxillin a.a. 1-151 used here). This suggests that in addition to motif preference, FAK phosphorylation might be affected by substrate conformation, specifically, by domains that affect substrate availability.

\section{FAK phosphorylation of CHUK/IKKa: role in NFKB signaling}

We identified four potential substrates involved in NFKB signaling: MYB binding protein 1a 
(MYBBP1A/p160) [15], conserved helix-loop-helix ubiquitous kinase (CHUK/IKKa) [16], TNFAIP3interacting protein 2 (TNIP2/Abin-2) [17], and TRAF2- and NCK-interacting kinase (TNIK) [18]. Previous studies have demonstrated a role for FAK kinase activity in NFKB signaling [19], including the ability of FAK to cooperate with NFkB to promote survival signaling [20]. Indeed, IPs containing HA-IKKa expressed in HEK293T cells could be tyrosine phosphorylated in FAK IVK assays (Fig. 4a), and as well, HA-IKKa could be tyrosine phosphorylated when co-expressed in HEK293T cells with a constitutively-active version of FAK, FAK180 [23] (Fig. 4b).

IKKa facilitates both canonical and non-canonical NFKB signaling (Fig. 4c), in the former case, by functioning within an IкB Kinase complex to directly phosphorylate the negative regulator, IкB [21], and in the latter case, by facilitating the cleavage p100 to produce a p52/RelB complex that controls the formation of tumor initiating cell subpopulations in breast cancer [22]. To dissect the role of FAK in NFKB signaling, we employed an NFKB-dependent luciferase reporter system. The level of NFkB activity was significantly decreased in FAK-null MEF (FAK-/-) as compared to MEF containing WT levels of FAK $(\mathrm{FAK}+/+)$ (Fig. 4d). Furthermore, re-expression of
FAK180 could partially rescue NFKB activity in FAK-/- MEF (Fig. 4e). In the human breast cancer cell line, MCF7, treatment with the FAK kinase inhibitor PF-573,228 (PF-228) specifically inhibited induction of canonical NFKB signaling by TNFa (Fig. 4f). FAK also plays a regulatory role in the non-canonical NFKB pathway because its transient overexpression in HEK293T cells induced the cleavage of p100 to p52 (Fig. 4g). Consistent with this role, treatment of HeLa cells with PF-228 prevented p100 cleavage mediated by the non-canonical pathway inducer, Lymphotoxin $\beta$ Receptor Ligand Light (heretofore called "Light") [24] (Fig. 4h). Lastly, in order to address how FAK phosphorylation of IKKa facilitates NFKB signaling, the major FAK phosphorylation sites on IKKa were identified as Y187 and Y198, based on anti-PTyr IB analysis of HEK293T cells co-transfected with IKKa WT or individual site $\mathrm{Y}-->\mathrm{F}$ variants plus FAK180 (Fig. 4i). In comparison to WT HA-IKKa or HA-IKKa ${ }^{500 F}$ (whose phosphorylation by FAK was not altered in Fig. 4i), HA-IKKa ${ }^{187 \mathrm{~F}}$ or HA-IKKa ${ }^{198 \mathrm{~F}}$ failed to induce NFKB signaling when transiently overexpressed in MCF-7 cells (Fig. 4j). Taken together, these data show that both canonical and non-canonical NFKB signaling are controlled by FAK, likely through the specific phosphorylation of IKKa.
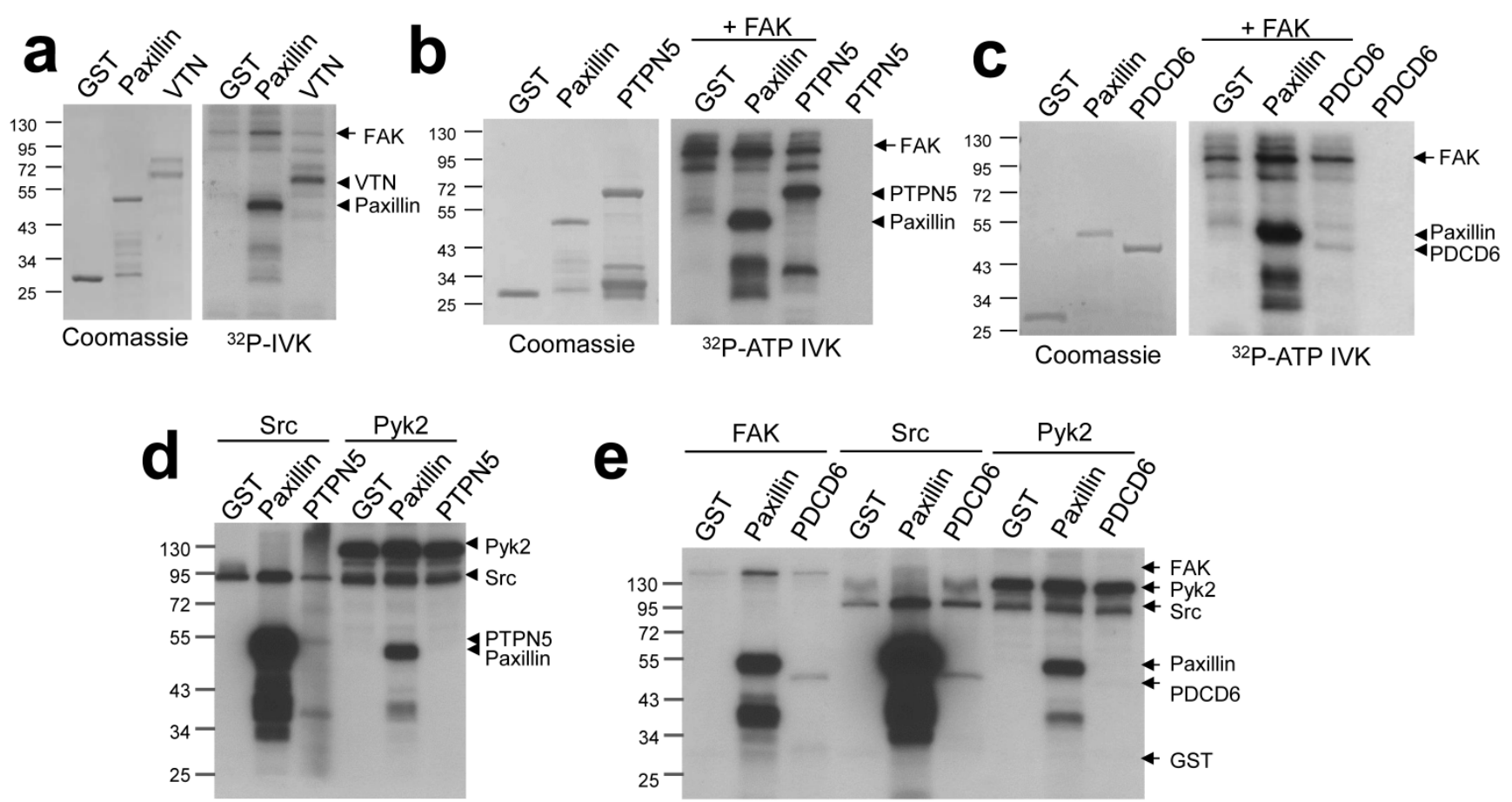

Figure 3. FAK phosphorylates vitronectin, PTPN5, and PDCD6. FAK-IVK reactions with VTN (a), GST-PTPN5 (b), GST-PDCD6 (c and e), using GST or GST-paxillin as negative or positive controls, respectively. Left panels (a-c): stained proteins; right panels: autoradiographs of FAK-IVK. d. IVK with purified Src or Pyk2 using GST, GST-paxillin, or GST-PTPN5 as substrates. e. IVK reactions with purified FAK, Src or Pyk2 using GST, GST-paxillin or GST-PDCD6 as substrates. 


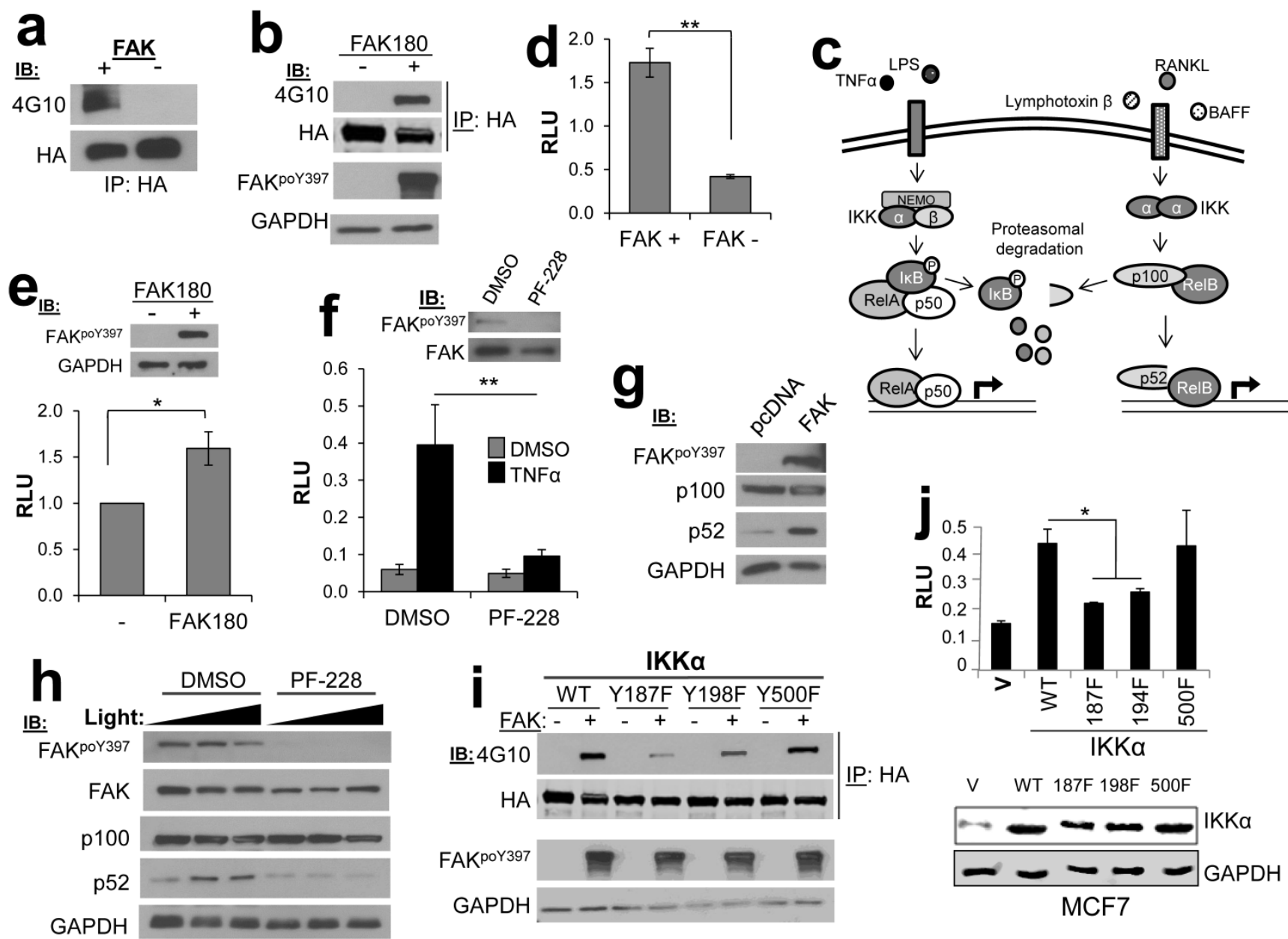

Figure 4. FAK phosphorylates CHUK/IKKa regulates NFKB signaling. a. FAK-IVK reaction using HA-IKKa IPs, identified by anti-PTyr IB. b. FAK180 phosphorylation of HA-IKKa in HEK293T cells. c. IKKa functions in both canonical (left side) and non-canonical (right side) NFKB pathways. d. Relative luciferase units (RLU) of NK-Luciferase reporter (normalized to CMV-Renilla) in FAK +/+ vs. FAK-/- MEF. e. RLU (NK-Luc vs CMV-Renilla) in FAK-/- cells transiently overexpressing FAK180. f. RLU (NK-Luc vs CMV-Renilla) in MCF7 cells stimulated with TNFa (20ng/mL for $16 \mathrm{~h}$ ). IB for active and total FAK (upper panel). g. FAK induces cleavage of p100 to p52 in HEK293T cells. h. p100 to p52 cleavage in HeLa cells ectopically expressing p100, after stimulation with 100 or $200 \mathrm{ng} / \mathrm{mL}$ Light for $16 \mathrm{~h}$ in the presence of either DMSO or $10 \mu \mathrm{M}$ PF-228. i. Decreased FAK180-induced $\mathrm{pT} \mathrm{Tr}$ of IKKa ${ }^{187 \mathrm{~F}}$ and IKKa ${ }^{198 \mathrm{~F}}$ relative to WT or IKKa ${ }^{500 F}$ in HEK293T cells ("-", vector). j. Decreased RLU (NK-Luc vs CMV-Renilla) in MCF7 transiently expressing IKKa ${ }^{187 F}$ and IKKa ${ }^{1985}$ relative to WT or IKKa ${ }^{500 F}(\mathrm{~V}$, vector). Error bars for all experiments: SEM, three independent experiments. *, $\mathrm{p}<0.05$; **, $\mathrm{p}<0.005$ as determined by unpaired $t$ test.

\section{Conclusion}

The in situ FAK kinase assay using protein microarrays is a novel and viable means to identify FAK substrates. Using this technology we have confirmed phosphorylation of four novel substrates of FAK, demonstrated specificity among FAK family members, and implicated FAK in regulation of two major signaling pathways. This screen represents a major starting point for an in-depth understanding of FAK's kinase activity and its role in tumorigenesis.

\section{Acknowledgements}

We thank Vita Golubovskaya, Xinjiang Wang, Andrei Gudkov, Andrei Bakin, Michael Schaller, Elena Kurenova for sharing reagents and cells. This work was supported by grants (I.H.G.) CA94108,
CA116430 (National Institutes of Health/National Cancer Institute), PC074228, PC101210 (Department of Defense), and in part, through National Cancer Institute Comprehensive Cancer funds (P30-CA016056).

\section{Conflict of Interest}

The authors have no conflicts of interest to report.

\section{References}

1. Hao H, Naomoto Y, Bao X, Watanabe N, Sakurama K, Noma K, et al. Focal adhesion kinase as potential target for cancer therapy (Review). Oncol Rep. 2009; 22(5): 973-9.

2. Schaller MD, Hildebrand JD, Shannon JD, Fox JW, Vines RR, Parsons JT. Autophosphorylation of the focal adhesion kinase, pp125FAK, directs SH2-dependent binding of pp60src. Mol Cell Biol. 1994; 14(3): 1680-8.

3. Calalb MB, Polte TR, Hanks SK. Tyrosine phosphorylation of focal adhesion kinase at sites in the catalytic domain regulates kinase activity: a role for Src family kinases. Mol Cell Biol. 1995; 15(2): 954-63. 
4. Owen JD, Ruest PJ, Fry DW, Hanks SK. Induced focal adhesion kinase (FAK) expression in FAK-null cells enhances cell spreading and migration requiring both auto- and activation loop phosphorylation sites and inhibits adhesion-dependent tyrosine phosphorylation of Pyk2. Mol Cell Biol. 1999; 19(7): 4806-18.

5. Bellis SL, Miller JT, Turner CE. Characterization of tyrosine phosphorylation of paxillin in vitro by focal adhesion kinase. J Biol Chem. 1995; 270(29): 17437-41.

6. Schlaepfer DD, Hunter T. Integrin signalling and tyrosine phosphorylation: just the FAKs? Trends Cell Biol. 1998; 8(4): 151-7.

7. Tachibana K, Urano T, Fujita H, Ohashi Y, Kamiguchi K, Iwata S, et al. Tyrosine phosphorylation of Crk-associated substrates by focal adhesion kinase. A putative mechanism for the integrin-mediated tyrosine phosphorylation of Crk-associated substrates. J Biol Chem. 1997; 272(46): 29083-90.

8. Ilic D, Furuta Y, Kanazawa S, Takeda N, Sobue K, Nakatsuji N, et al. Reduced cell motility and enhanced focal adhesion contact formation in cells from FAK-deficient mice. Nature. 1995; 377(6549): 539-44.

9. Sieg DJ, Ilic D, Jones KC, Damsky CH, Hunter T, Schlaepfer DD. Pyk2 and Src-family protein-tyrosine kinases compensate for the loss of FAK in fibronectin-stimulated signaling events but Pyk2 does not fully function to enhance FAK- cell migration. EMBO J. 1998; 17(20): 5933-47.

10. Lin X, Tombler E, Nelson PJ, Ross M, Gelman IH. A novel src- and ras-suppressed protein kinase $\mathrm{C}$ substrate associated with cytoskeletal architecture. J Biol Chem. 1996; 271(45): 28430-8.

11. Sonoda Y, Watanabe S, Matsumoto Y, Aizu-Yokota E, Kasahara T. FAK is the upstream signal protein of the phosphatidylinositol 3-kinase-Akt survival pathway in hydrogen peroxide-induced apoptosis of a human glioblastoma cell line. J Biol Chem. 1999; 274(15): 10566-70.

12. Schlaepfer DD, Jones KC, Hunter T. Multiple Grb2-mediated integrin-stimulated signaling pathways to ERK2/mitogen-activated protein kinase: summation of both c-Src- and focal adhesion kinase-initiated tyrosine phosphorylation events. Molec Cell Biol. 1998; 18(5): 2571-85.

13. Fox JL, Ismail F, Azad A, Ternette N, Leverrier S, Edelmann MJ, et al. Tyrosine dephosphorylation is required for Bak activation in apoptosis. EMBO J. 2010; 29(22): 3853-68

14. Huang Y, Jin H, Liu Y, Zhou J, Ding J, Cheng KW, et al. FSH inhibits ovarian cancer cell apoptosis by up-regulating survivin and down-regulating PDCD6 and DR5. Endocr Relat Cancer. 2011; 18(1): 13-26.

15. Owen HR, Elser M, Cheung E, Gersbach M, Kraus WL, Hottiger MO. MYBBP1a is a novel repressor of NF-kappaB. J Mol Biol. 2007; 366(3): 725-36.

16. DiDonato JA, Hayakawa M, Rothwarf DM, Zandi E, Karin M. A cytokine-responsive IkappaB kinase that activates the transcription factor NF-kappaB. Nature. 1997; 388(6642): 548-54.

17. Leotoing L, Chereau F, Baron S, Hube F, Valencia HJ, Bordereaux D, et al. A20-binding inhibitor of nuclear factor-kappaB (NF-kappaB)-2 (ABIN-2) is an activator of inhibitor of NF-kappaB (IkappaB) kinase alpha (IKKalpha)-mediated NF-kappaB transcriptional activity. J Biol Chem. 2011; 286(37): 32277-88.

18. Shkoda A, Town JA, Griese J, Romio M, Sarioglu H, Knofel T, et al. The germinal center kinase TNIK is required for canonical NF-kappaB and JNK signaling in B-cells by the EBV oncoprotein LMP1 and the CD40 receptor. PLoS Biol. 2012; 10(8) :e1001376.

19. Funakoshi-Tago M, Sonoda $Y$, Tanaka S, Hashimoto K, Tago K, Tominaga S, et al. Tumor necrosis factor-induced nuclear factor kappaB activation is impaired in focal adhesion kinase-deficient fibroblasts. J Biol Chem. 2003; 278(31): 29359-65.

20. Zhang HM, Keledjian KM, Rao JN, Zou T, Liu L, Marasa BS, et al. Induced focal adhesion kinase expression suppresses apoptosis by activating NF-kappaB signaling in intestinal epithelial cells. Am J Physiol Cell Physiol. 2006; 290(5): C1310-20.

21. Karin M, Yamamoto Y, Wang QM. The IKK NF-kappa B system: a treasure trove for drug development. Nat Rev Drug Discov 2004; 3(1): 17-26.

22. Kendellen MF, Bradford JW, Lawrence CL, Clark KS, Baldwin AS. Canonical and non-canonical NF-kappaB signaling promotes breast cancer tumor-initiating cells. Oncogene. 2014; 33(10): 1297-305.

23. Lietha D, Cai X, Ceccarelli DF, Li Y, Schaller MD, Eck MJ. Structural basis for the autoinhibition of focal adhesion kinase. Cell. 2007; 129(6): 1177-87.

24. Mordmuller B, Krappmann D, Esen M, Wegener E, Scheidereit C. Lymphotoxin and lipopolysaccharide induce NF-kappaB-p52 generation by a co-translational mechanism. EMBO Rep. 2003; 4(1): 82-7. 